\title{
Operational infrastructure to ensure the long-term sustainability of the IHRS/IHRF
}

${ }^{1}$ Riccardo Barzaghi, 2Laura Sánchez, ${ }^{3}$ George Vergos

${ }^{1}$ Politecnico di Milano, Italy

2Deutsches Geodätisches Forschungsinstitut, Technische Universität München, (DGFI-TUM), Germany

${ }^{3}$ Department of Geodesy and Surveying, Aristotle University of Thessaloniki, Greece

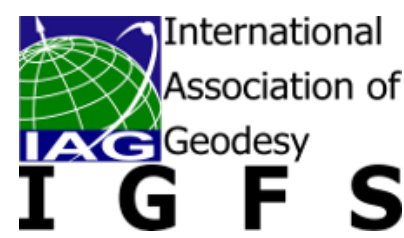

EGU2020 General Assembly

Vienna, Austria, May 4-8, 2020 


\section{Motivation}

The International Height Reference System (IHRS) is based on the combination of

- a geometric component given by the coordinate vector $\mathbf{X}$ in the ITRS and

- a physical component given by the determination of potential values $\mathrm{W}$ at $\mathrm{X}$.

The determination of $X$

- is performed following the standards and conventions of the IERS

- is supported by a strong operational infrastructure (reference stations, data centres, analysis centres, combination centres, product centres, etc.) acting under the responsibility of the geometric IAG services

- is based on an experience larger than 30 years (first realisation of the ITRS in 1989 - ITRF89).

A similar foundation is needed for the reliable and long-term sustainable realisation of the IHRS: the International Height Reference Frame (IHRF). 


\section{Objectives}

- To identify the elements needed to install an operational infrastructure for the IHRS/IHRF similar to the infrastructure existing for the ITRS/ITRF.

- To describe how the existing gravity field-related IAG services could contribute to the IHRS/ IHRF.

- To identify missing elements and interfaces to be established within the International Gravity Field Service (IGFS) as "natural home" of the IHRS/ITRF. 


\section{Reference network}

ITRS/ITRF

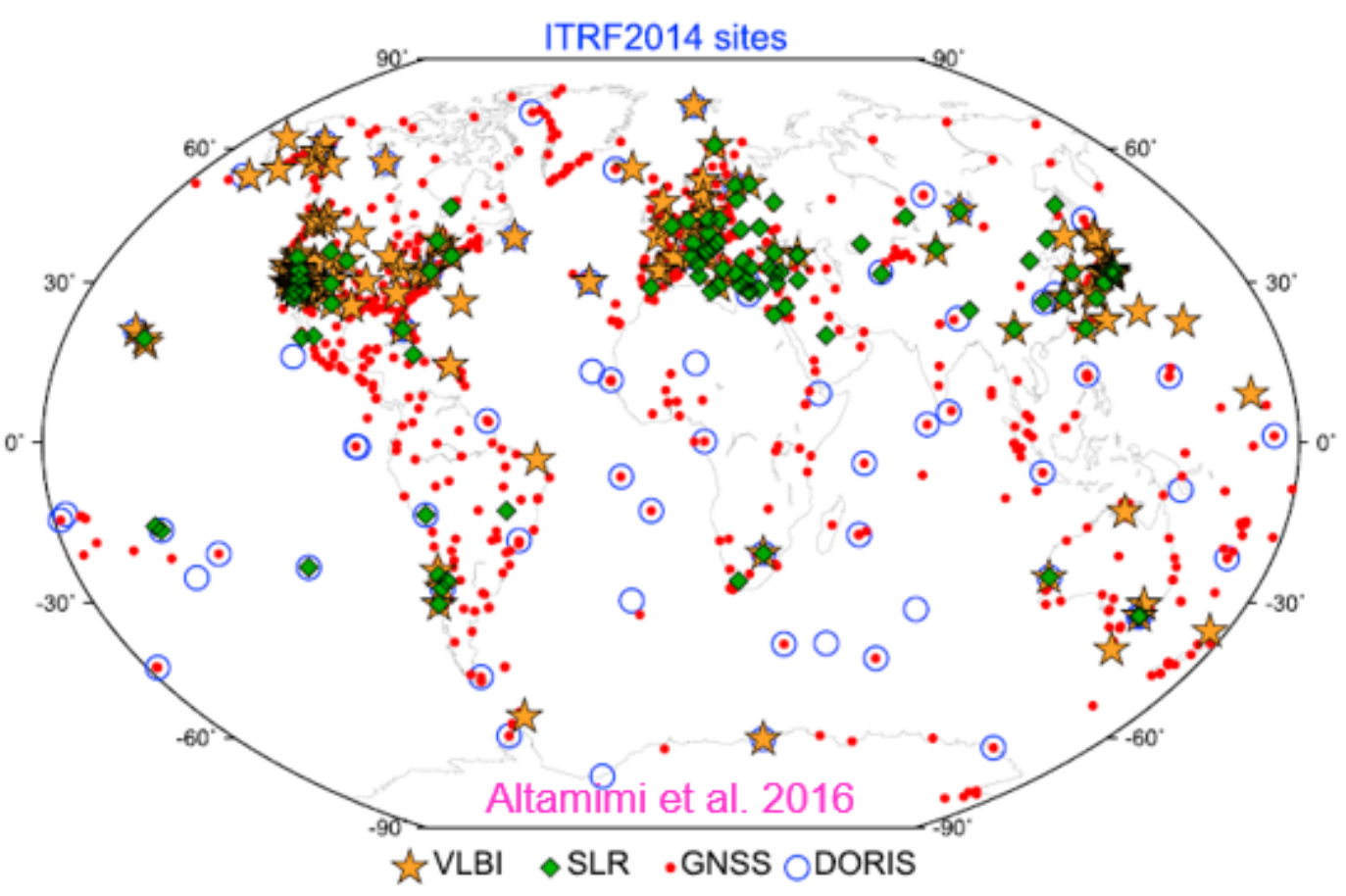

- Expensive techniques (VLBI, SLR, DORIS) supported by space and geodetic agencies.

- Densification with GNSS based on contributions from the regional and national reference frames.

- Station selection in accordance to the station performance and quality (IVS, ILRS, IGS, IDS).

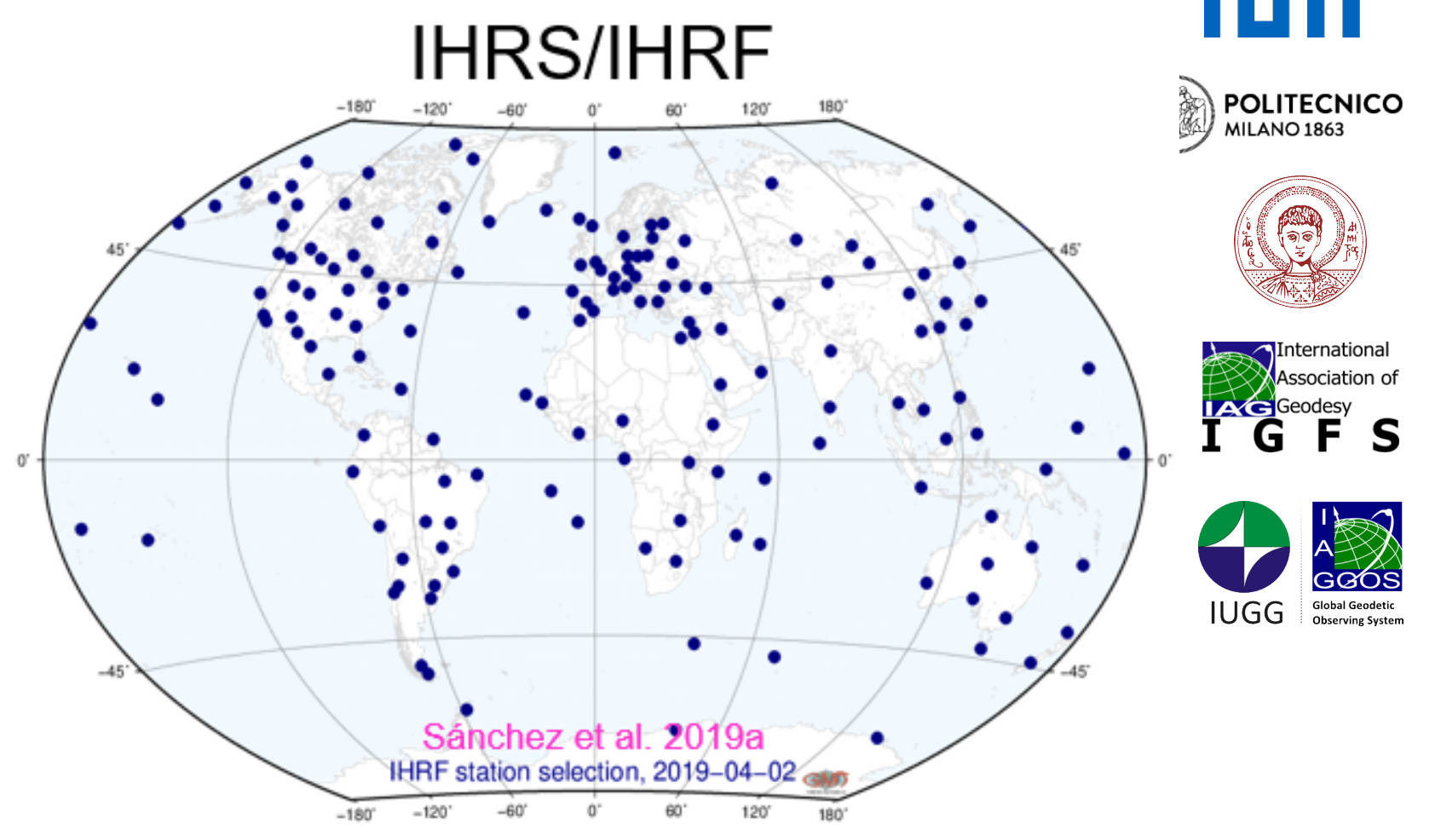

- Primary realisation with GNSS and co-located with VLBI, SLR, DORIS, absolute gravity, tide gauges and levelling networks.

- Gravity data of high resolution for reliable determination of W.

- Densification based on the recommendations from the regional and national reference frames.

- Quality of X assessable, quality of W usually not checked 


\section{Input data and data flow}

\section{ITRS/ITRF}
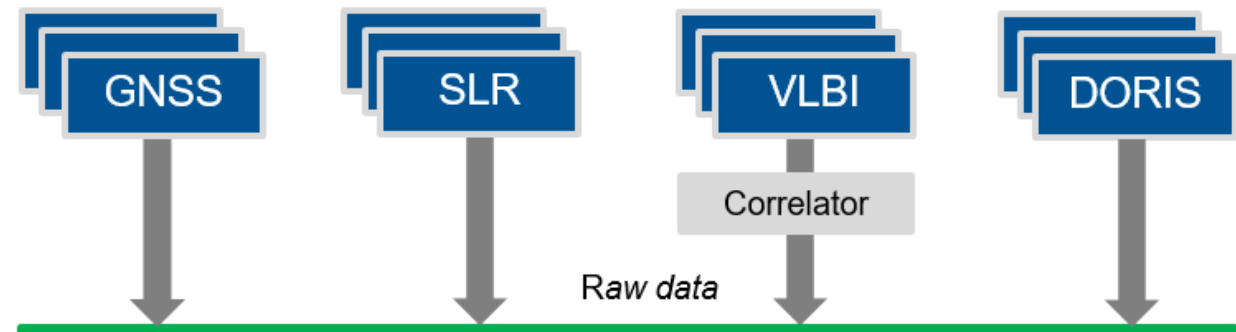

Operational / Regional Data Centers

\section{Data}

Global Data Centers

Data

Service Products

Analysis and Combination Centers

Products, Metadata

Archives and User Access (IAG products are freely available)

Angermann et al. 2019

\section{IHRS/IHRF}
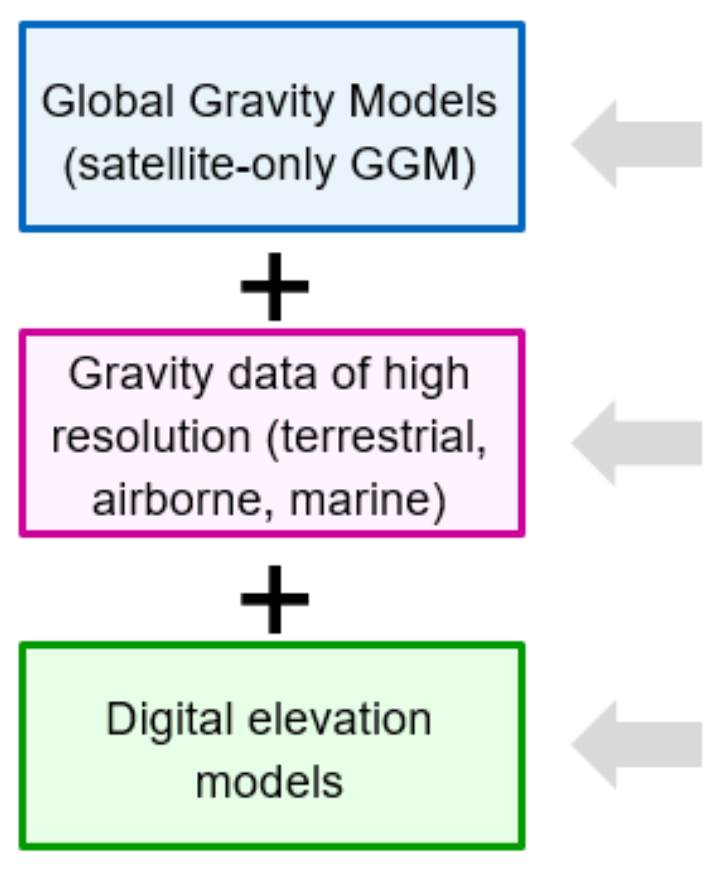

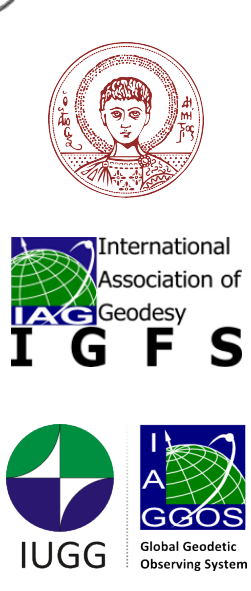

International Center for Global Earth Models

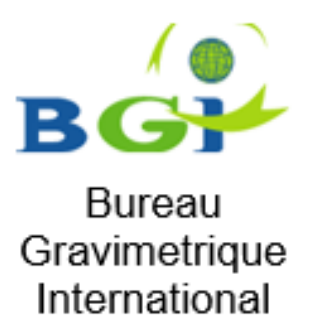

$$
\begin{gathered}
\text { International Digital } \\
\text { Elevation Model Service }
\end{gathered}
$$

- ICGEM: data freely available, quality assessment

- BGI: historical gravity data freely available but uncertainty about reference system for gravity and positions, low accuracy. Recent gravity data with restricted access.

- IDEMS: elevation models of low resolution and accuracy freely available, better elevation models with costs. Softwaredependent accessibility. 


\section{Organisational scheme for computation}

ITRS/ITRF

Geometric IAG Services

Position and EOP time series (daily / weekly)

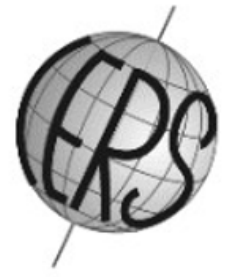

\section{IHRS/IHRF}

- A centralised computation of $\mathrm{W}$ is (still) unviable due to the restricted accessibility to terrestrial gravity data and elevation models of high-resolution.

- To exploit at maximum the existing data to get as accurate as possible potential values, national/regional experts in the gravity field (or geoid) modelling should be involved in the determination of the IHRS/IHRF coordinates.

- A rigorous standardisation seems to be not suitable because

1) it exists different data availability and different data quality around the world and

- Consistency trough IERS standards and conventions

- Centralised estimation by combining the technique-based solutions at the combination centres

- Redundancy: three independent combinations.
2) regions with different characteristics require particular approaches

- Quality assessment of the individual computations is very difficult because redundancy is difficult to require.
POLITECNICO MILANO 1863

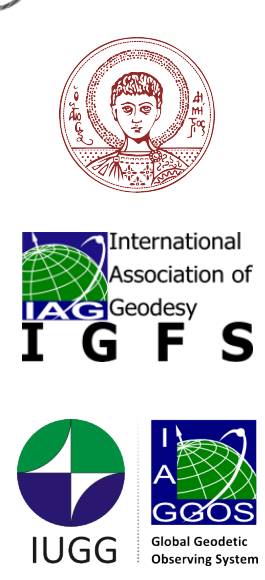




\section{Usability}

\section{ITRS/ITRF}

- In scientific applications, the ITRF is the best available platform for the determination of geometric coordinates (positions, trajectories, velocities). It is well accepted and widely used.

- In practical and non-geodetic applications, the ITRF is "involuntarily" accepted and used by everyone using GNSS techniques and ITRFbased satellite orbits.

- Regional and national densifications of the ITRF have been necessarily established, because GNSS is the primary technique used today for the establishment of reference frames.

\section{IHRS/IHRF}

- Discussions about a world height system or a global vertical reference system started 30 years ago. Realisation for the first time possible after GRACE and GOCE. However, accuracy one to two levels worse than by the ITRF.

- To achieve a wide acceptance of the IHRS/IHRF, the certainty of potential values (gravity field modelling at high resolution) has to be drastically improved.

- In the best case, the IHRF will be "involuntarily" accepted and used by everyone using GNSS in combination with a consistent geopotential model of high resolution.

- Most probably, the existing height systems will continue in daily use and the IHRS/IHRF will be considered for selected applications only. 


\section{Recommendations}

- To increase the reliability of the potential values the old gravity data sets have to be replaced with modern gravity data: referring to the Absolute Gravity Reference Frame (IGRF), with precise ( 2 $\mathrm{cm}$ ) 3D coordinates referring to the ITRF, and a high spatial resolution (ideal 3 to $5 \mathrm{~km}$ ). The IGFS is not able to pay for gravity surveys, but its support is indispensable by providing

$\rightarrow$ a complete inventory of the existing absolute gravity stations (as at present BGI does)

$\rightarrow$ guidelines for measuring and processing absolute and relative terrestrial gravity observations and airborne (marine) gravity surveys.

- The commercial (private) character of many gravity data sets has been a point of discussion over many years. The IGFS cannot obligate anybody to make freely available private gravity data, but it can

$\rightarrow$ outline political and technical arguments oriented to convince policy makers about the necessity of getting access to these data at national level for the gravity field (geoid) modelling. A direct link to the UN Resolution of the GGRF would be useful. 


\section{Recommendations}

- National/regional experts on gravity field modelling should be involved in the determination of the IHRS/ IHRF coordinates. They should utilise all the data they have available to determine the potential values using the computation approaches they have implemented for their regions. To minimise discrepancies and to obtain as similar and compatible results as possible

$\rightarrow$ a set of basic standards should be defined and updated in accordance with new models and procedures (start point: outcomes of the Colorado experiment)

- To assess the reliability of the individual computations performed by the national/regional experts

$\rightarrow 1$ ) "Auxiliary" stations close (up to $\sim 100 \mathrm{~km}$ ) to the core IHRF stations connected by levelling of high precision to have independent data (potential differences) for evaluation;

$\rightarrow 2$ ) Redundancy in the computation should be ensured: an IGFS "analysis centre" or IGFS "associates" could be provided with local data to determine potential values to be compared with the local results, or

$\rightarrow 3$ ) Computation methods used by national/regional experts should be calibrated: determination of potential values using a certain set of input data and comparison with results obtained by other approaches (like in the Colorado experiment) 


\section{Recommendations}

- To keep the IHRS realisation up-to-date, it is necessary

$\rightarrow$ The interaction with the GGOS Bureau for Networks and Observations, the IERS (ITRF reference network), the BGI (IGRF network), and national/regional experts to maintain the reference network

$\rightarrow$ Regular updates of the IHRFyyyy (synchronised with the release of new ITRF solutions) to take account for:

- new stations;

- coordinate changes with time $\dot{\mathrm{X}}, \dot{\mathrm{W}}$;

- improvements in the estimation of X and W (new observations, better standards, better models, better computation algorithms, etc.). 


\section{Summary: IHRF "element" within the IGFS}
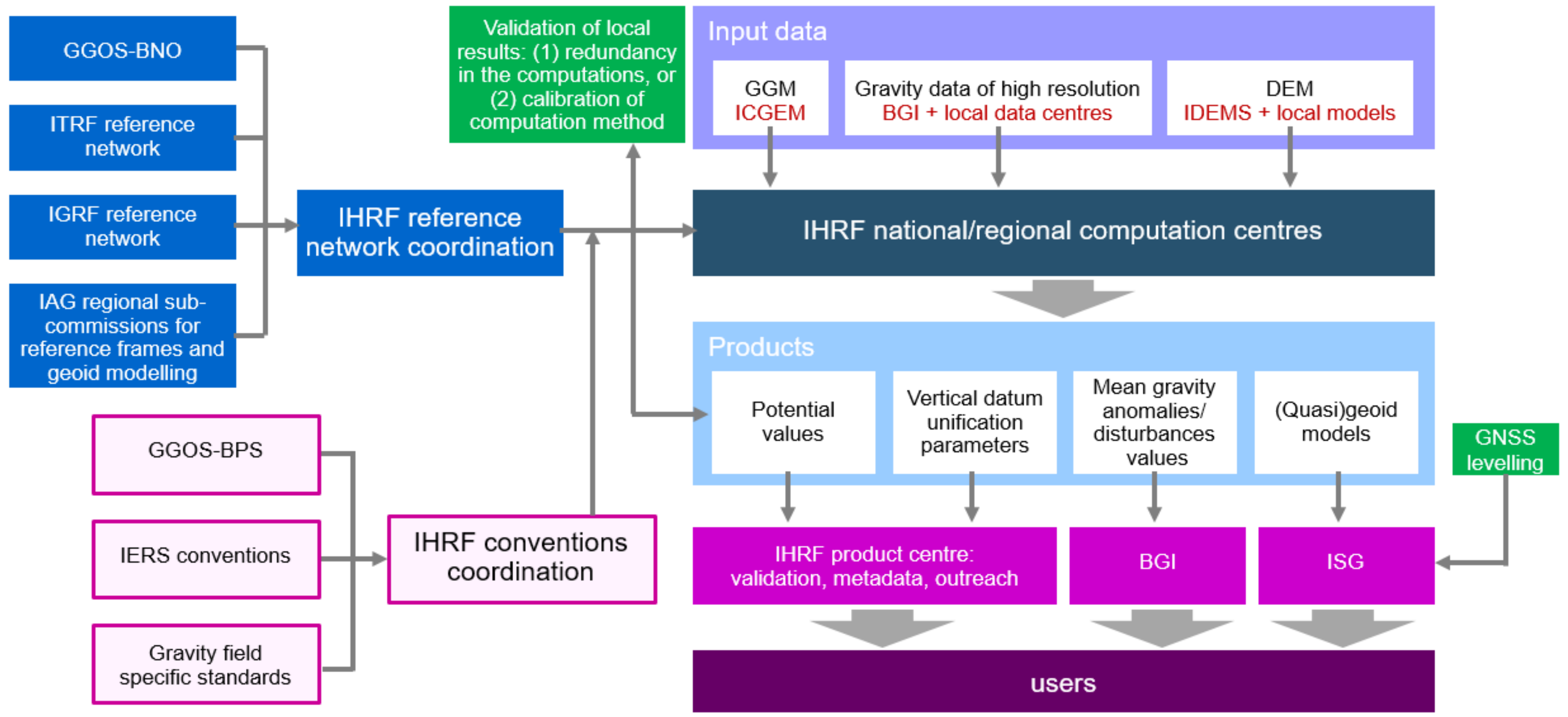

Sánchez et al. (2019b) 


\section{More details about the implementation of the IHRS/IHRF can be found at:}

Sánchez L (2019). Report of the GGOS Focus Area "Unified Height System" and the Joint Working Group 0.1.2: Strategy for the Realization of the International Height Reference System (IHRS), Reports 2015-2019 of the International Association of Geodesy (IAG), Travaux de l'AIG Vol. 41: 583-592.

Sánchez L, et al. (2019a). Advances in the realisation of the International Height Reference System. GGOS Days 2019 and Symposium SIRGAS2019, Rio de Janeiro, Brazil. Nov 11 - 14, 2019, http://www.sirgas.org/fileadmin/docs/Boletines/Bol24/42_Sanchez_et_al_2019_IHRS_IHRF_advances.pdf.

Sánchez L, et al. (2019b). Operational infrastructure to ensure the long-term sustainability of the IHRS/IHRF. IUGG General Assembly 2019, Montreal, Canada. Available at https://mediatum.ub.tum.de/doc/1537756/1537756.pdf. Last accessed 2020-04-13.

Sánchez L., Barzaghi R (2020). Activities and plans of the GGOS Focus Area Unified Height System, EGU General Assembly 2020, EGU2020-8625, https://doi.org/10.5194/egusphere-egu2020-8625, https://presentations.copernicus.org/EGU2020/EGU2020-8625_presentation.pdf 